\title{
Thickness and component distributions of yttrium-titanium alloy films in electron- beam physical vapor deposition
}

\author{
LI ShuaiHui, SHU YongHua \& FAN Jing ${ }^{\dagger}$ \\ Laboratory of High Temperature Gas Dynamics, Institute of Mechanics, Chinese Academy of Sciences, \\ Beijing 100190, China
}

Thickness and component distributions of large-area thin films are an issue of international concern in the field of material processing. The present work employs experiments and direct simulation Monte Carlo (DSMC) method to investigate three-dimensional low-density, non-equilibrium jets of yttrium and titanium vapor atoms in an electron-beams physical vapor deposition (EBPVD) system furnished with two or three electron-beams, and obtains their deposition thickness and component distributions onto 4 -inch and 6 -inch mono-crystal silicon wafers. The DSMC results are found in excellent agreement with our measurements, such as evaporation rates of yttrium and titanium measured in-situ by quartz crystal resonators, deposited film thickness distribution measured by Rutherford backscattering spectrometer (RBS) and surface profilometer and deposited film molar ratio distribution measured by RBS and inductively coupled plasma atomic emission spectrometer (ICP-AES). This can be taken as an indication that a combination of DSMC method with elaborate measurements may be satisfactory for predicting and designing accurately the transport process of EBPVD at the atomic level.

electron beam physical vapor deposition, thin film, thickness and species distributions, vapor atom, nonequilibrium transport

It is very important to predict and control the transport process of vapor atoms from evaporation sources to substrates in physical vapor deposition, particularly for fabrication of large-area, multi-component thin films. The transport process affects significantly the film thickness and component distributions, and thus plays a crucial role for really achieving material design and fabrication at the atomic level.

This article is mainly devoted to the interaction between three dimensional low-density, non-equilibrium vapor jets in a multi-electron-beam physical vapor deposition (EBPVD) system,

Received January 23, 2007; accepted May 11, 2007 doi: 10.1007/s11431-008-0096-7

${ }^{\dagger}$ Corresponding author (email: jfan@imech.ac.cn)

Supported by the National Natural Science Foundation of China (Grant Nos. 90205024,10502051 and 10621202) 
with emphasis laid on the particle fluxes impinging upon the substrate. EBPVD is a common method to fabricate thin films in vacuum that utilizes high-speed electrons to strike an ingot in a water-cooled crucible. The ingot absorbs the electron energy, resulting in a rapid rise of temperature at its surface. The atoms evaporating from the surface expand in vacuum and impinge upon a substrate, and then a film will grow on its surface under appropriate conditions ${ }^{[1]}$.

The early models of the vapor particle flux on a substrate in EBPVD were based on the free molecular assumption ${ }^{[2,3]}$. For a planar evaporation source, the particles will fly to the substrate along a straight-line path when the collisions between them are ignored. Consequently, the particle flux on a substrate element with the area of $A_{s}$ is

$$
q_{s}=\frac{A_{s} \cos \phi \cos \varphi}{\pi r^{2}} q_{e} .
$$

The cosine law in eq. (1) is intuitive and simple. However, experiments ${ }^{[4,5]}$ showed that $q_{s}$ was usually proportional to $\cos ^{n} \varphi$, while the value of $n$ was dependent on the Knudsen number $(K n)$ of the vapor jet.

The Knudsen number of vapor jets in EBPVD is often varying tremendously in the flow field. For instance, in EBPVD of yttrium investigated in ref. [6], the local Knudsen number at the evaporation source was 0.8 at the evaporation source, and increased to $10^{2}$ at the substrate that was only 15 inch away from the source in the normal direction (Figure 7 in ref. [6]).

In the transitional regime $(0.1<K n<10)$, the traditional continuum assumption and free molecular approximation are no longer valid, and currently the most powerful analytical tool is the direct simulation Monte Carlo (DSMC) method ${ }^{[7,8]}$. A critical step in the DSMC method is to determine the collision cross section between particles. Phenomenological model $\mathrm{s}^{[9-13]}$ based on the Chapman-Enskog theory ${ }^{[14]}$ are often used to obtain molecular and atomic collision cross sections from experimental data of transport coefficients that are available for many common gases such as helium, argon, nitrogen, oxygen, etc. However, for metal vapors of interests in EBPVD, such as yttrium, titanium and copper, measurement data of transport coefficients are highly insufficient. To overcome the difficulty, Fan et al. ${ }^{[6]}$ made use of the viscosity coefficients of the alkali metal vapors to determine their collision parameters in the variable hard-sphere (VHS) model commonly used in DSMC, and then extrapolated them to other metal atoms by referring to the periodic table of elements. This approach was experimentally validated in various DSMC calculations of vapor deposition of yttrium ${ }^{[6]}$, titanium ${ }^{[15]}$ and aluminum ${ }^{[16]}$.

For large-area film co-deposition with multi-sources, the interaction between different vapor jets has to be taken into account. Monte Carlo modeling of YBCO vapor deposition ${ }^{[17]}$ showed that the atomic collisions between different components seriously affected the molar ratio of components in the deposited film as the evaporation rates increased, and even made them deviate completely from the ratios of the corresponding source's evaporation rates.

The DSMC method and experiments are used here to study a three-dimensional low-density, non-equilibrium flow field of mixed vapor atoms in an EBPVD system furnished with two or three evaporation sources, as well as the thickness and component distributions of the deposited films of yttrium-titanium alloy. First, an experimental facility of EBPVD and related measurement methods are briefly described, followed by an illustration of the computational conditions for DSMC. Then the DSMC and expimental results are presented and compared, and conclusions are drawn in the last section. 


\section{Experimental facility and measurement methods}

\subsection{EBPVD facility}

As shown in Figure 1, our experimental facility of EBPVD is named as RGD-3 $3^{[18]}$ and its main body is a cylindrical vacuum chamber with a diameter of $760 \mathrm{~mm}$, height of $850 \mathrm{~mm}$, and water cooled side-wall. There are three sets of electron guns and evaporation crucibles and one set of electric resistance evaporation equipment inside. The rate of each evaporation source is monitored and controlled by the corresponding quartz crystal probe.

Three sets of compound vacuum gauges are installed on the side-wall and top flange of the vacuum chamber, respectively, to measure the pressures at the different locations. The background pressure in the chamber during a film deposition process depends upon the outgassing characteristics and evaporation rates of evaporative materials, the flux of in-situ annealing gases, and so on. Generally it keeps at $1 \times 10^{-3} \mathrm{~Pa}$ or so.

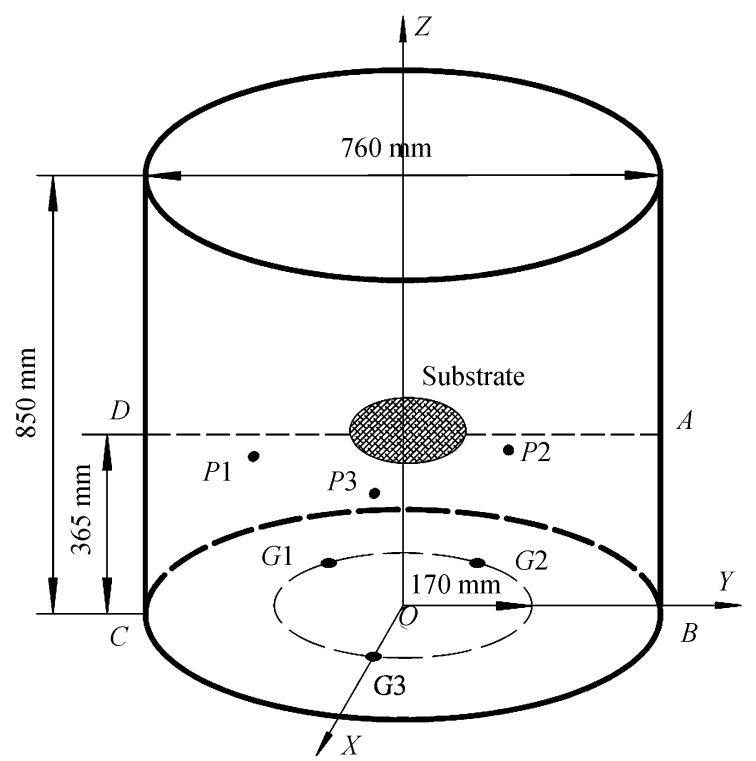

Figure 1 Schematic diagram of the positions of evaporation sources, quartz crystal probes, and substrate in the vacuum chamber of RGD-3.

\subsection{Experimental conditions}

Experiments of co-depositing yttrium-titanium alloy film are carried out using two and three evaporation sources in RGD-3, respectively, and the experimental conditions are given in Table 1. In a Cartesian system with its origin $O$ at the center of the chamber bottom, three evaporation sources $G 1-G 3$ are uniformly distributed over a circle with a radius of $170 \mathrm{~mm}$ and centered at $O$. The $X$ axis passes through the center of $G 3$, and the $Y$ axis is parallel to the line connecting the centers of $G 1$ and $G 2$.

Mono-crystal silicon wafers are used in both experiments, with diameters of 4 inches and 6 inches, respectively. The vertical distance from the substrate to the evaporation source plane is 365 $\mathrm{mm}$. During the experiments, the substrates are kept at the room temperature, and the evaporative times last $1000 \mathrm{~s}$ and $780 \mathrm{~s}$, respectively.

Yttrium and titanium ingots with even and non-oxidative surfaces are cleaned by acetone and 
alcohol, weighed with an electronic microbalance, and then put in the water-cooled crucibles. After the experiments, the electronic microbalance is used again to weigh the post-evaporation masses of the yttrium and titanium ingots taken from the crucibles, and a vernier caliper is used to measure the diameter of the evaporative area $d_{e}$. The evaporation mass $M_{e}$ is obtained by comparing the preand post-evaporation masses.

It is easy to calculate the evaporation rate when the evaporation mass, time and area are known, i.e.

$$
\Gamma_{e}=\frac{4 M_{e}}{\pi d_{e}^{2} t_{e}} .
$$

Dushman ${ }^{[19]}$ provided the relation of $\Gamma_{e}$ to the evaporation surface temperature $T_{e}$ as follows:

$$
\log \Gamma_{e}=C-0.5 \log T_{e}-B / T_{e} .
$$

The fitting values of $B$ and $C$ for common metals may be found in Table 10.2 of ref. [19], which are $2.197 \times 10^{4}$ and 9.17 for yttrium, and $2.323 \times 10^{4}$ and 9.11 for titanium, respectively.

Substitution of the evaporation rates under the experimental conditions into eq. (3) gives $T_{e}$ (Table 1). According to the kinetic theory ${ }^{[7,8]}$, the number density at the evaporation source surface is

$$
n_{e}=\Gamma_{e} \sqrt{2 \pi / k m T_{e}}
$$

\begin{tabular}{|c|c|c|c|c|c|c|}
\hline Case & $\begin{array}{c}\text { Evaporation } \\
\text { source }\end{array}$ & $\begin{array}{c}\text { Evaporation } \\
\text { materials }\end{array}$ & $M_{e}(\mathrm{~g})$ & $d_{e}(\mathrm{~cm})$ & $T_{e}(\mathrm{~K})$ & $n_{e}\left(\times 10^{19} \mathrm{~m}^{-3}\right)$ \\
\hline \multirow{2}{*}{ I } & $G 1$ & $\mathrm{Ti}$ & 0.289 & 1.2 & 2099 & 12.6 \\
\hline & $G 2$ & $\mathrm{Yt}$ & 0.232 & 3.0 & 1887 & 3.1 \\
\hline \multirow{3}{*}{ II } & $G 1$ & $\mathrm{Yt}$ & 0.187 & 1.2 & 1957 & 7.9 \\
\hline & $G 2$ & $\mathrm{Yt}$ & 0.217 & 1.2 & 1969 & 9.2 \\
\hline & G3 & $\mathrm{Ti}$ & 0.193 & 1.2 & 2086 & 10.8 \\
\hline
\end{tabular}

where $k$ is the Boltzmann constant, and $m$ is the atomic mass.

Table 1 Experimental conditions in co-deposition of yttrium-titanium alloy films

\subsection{Measurement of evaporation rate}

The evaporation rates of $G 1-G 3$ are in-situ monitored and controlled by quartz crystal resonator probes $P 1-P 3$ made by Inficon, respectively. In order to shield the disturbances from other evaporation sources, each probe is surrounded with a rectangular shelter.

The core part of the probes $P 1-P 3$ is a quartz crystal slice cut at certain azimuth angle. The top and bottom surfaces of the slice that are partially coated with a gold or silver film are used as electrodes. Under an external alternating current frequency, the amplitude of the quartz crystal slice increases significantly, while the piezoelectric resonance frequency is rather sensitive to the deposition thickness of vapor particles $\underline{[20]}$.

Table 2 gives the space coordinates of $P 1$ and $P 2$, as well as the components of the normal direction of the quartz crystal slice $\left(l_{X}, m_{Y}, n_{Z}\right)$. Figure 2 shows the measured curves of the deposition rates $E_{s}$ onto the quartz crystal resonance surfaces of $P 1$ and $P 2$ in Case I. For a practical process, it is of considerable interest to establish relationship between these measured curves and the evaporation rates of $G 1$ and $G 2$. Such a relation is built here based on rarefied vapor flow fields calculated by the DSMC method, which agrees quite well with the experimental data. Refer to Section 3.1 for detail. 
Table 2 Positions and unit vectors normal to the surfaces of the quartz crystal probes $P 1$ and $P 2$ in RGD-3

\begin{tabular}{ccccccc}
\hline Resonator & $X(\mathrm{~mm})$ & $Y(\mathrm{~mm})$ & $Z(\mathrm{~mm})$ & $l_{X}$ & $m_{Y}$ & $n_{Z}$ \\
\hline$P 1$ & -250 & -244 & 260 & 0.208 & -0.342 & 0.921 \\
$P 2$ & -300 & 220 & 230 & 0.225 & 0.259 & 0.940 \\
\hline
\end{tabular}

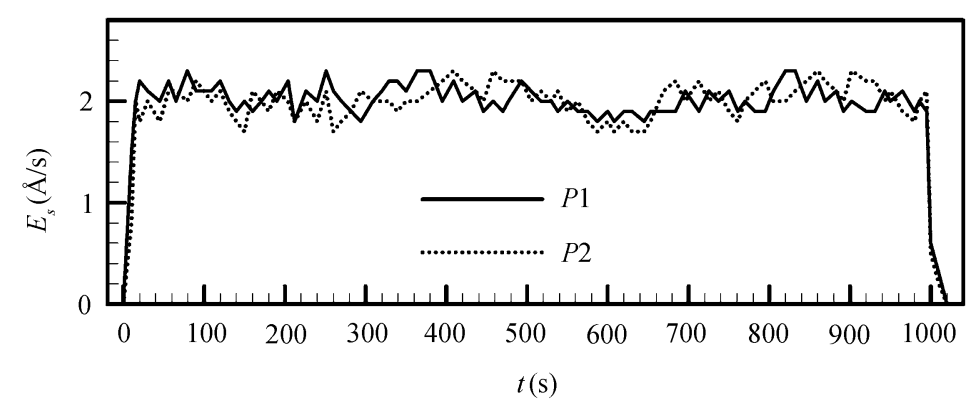

Figure 2 Measured curves of yttrium and titanium deposition rates versus time given by $P 1$ and $P 2$ for Case I.

\subsection{Measurement of film thickness}

For comparison and verification, two different methods are used respectively to measure the film thickness and the molar ratio of yttrium and titanium. For the former, a surface profilometer and a Rutherford backscattering spectrometer (RBS) are employed; and for the latter, RBS and an inductively coupled plasma atomic emission spectrometer (ICP-AES) are adopted. Because the surface profilometer and RBS measurements do not damage a film sample, we measure firstly its thickness with the surface profilometer, then its thickness and molar ratio with RBS, and finally its molar ratio with ICP-AES.

A Dektak8 type of surface profilometer is used in our measurement. A silicon substrate is partially shielded by smooth strip-type slices of $0.5 \mathrm{~mm}$ thickness during deposition. The slices are taken off after experiments, and then proper film steps would appear that are suitable for Dektak8 to measure. Figure 3 shows the film step distribution and measured data of thickness in Case II.

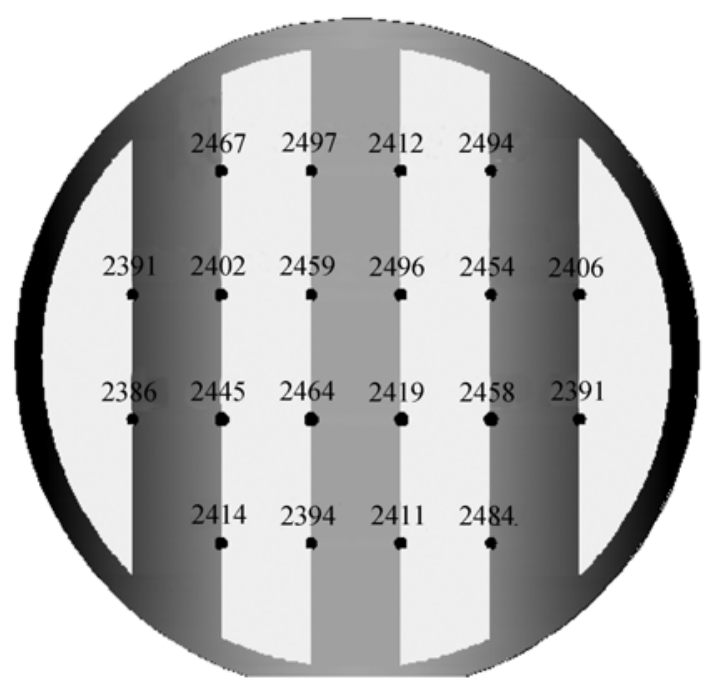

Figure 3 Yttrium-titanium alloy film thickness distribution over a 6-inch silicon wafer measured with a surface profilometer in Case II (unit: $\AA$ ). 
A 5SDH-2 type RBS made by NEC is used in our measurement. Its incident alpha particles have an energy $E_{0}=2022 \mathrm{keV}$, mass $m_{\alpha}=4 \mathrm{amu}$ and charge number $Z_{\alpha}=2$. The angle between the incident particle beam and the normal direction of the 5SDH-2 probe is $\vartheta=165^{\circ}$.

For a yttrium and titanium film studied here, the scattering energies of $\alpha$ particles after colliding with the nuclei of component $j(j=1,2)$ at the front and back surfaces of the film are denoted as $E_{j, a}$ and $E_{j, b}$, respectively, which correspond to the front and back edges of the characteristic peak of component $j$ in an energy spectrum of RBS. Such a backscattering process may be described by the hard-sphere collision dynamics ${ }^{[21]}$, and therefore

$$
K_{j} \equiv \frac{E_{j, a}}{E_{0}}=\left(\frac{\sqrt{m_{j}^{2}-m_{\alpha}^{2} \sin ^{2} \vartheta}+m_{\alpha} \cos \vartheta}{m_{\alpha}+m_{j}}\right)^{2} .
$$

Substituting the measured value of $E_{j, a}$ into eq. (5), we obtain $m_{j}$, from which the element type is determined by referring to the periodic table of elements.

Using the measured data of $E_{j, a}$ and $E_{j, b}$, together with the energy loss factors $\varepsilon_{i}$ and $\varepsilon_{o}$ of $\alpha$ particles during an incident and scattering process calibrated by experiment ${ }^{[22]}$, the atomic surface density of a film can be calculated as follows:

$$
\xi=\Delta E_{j} /\left(K_{j} \varepsilon_{i}+\varepsilon_{o}\right) .
$$

If the volume of an atom in the film is assumed to be $m_{j} / \rho_{j}$, then the film thickness is

$$
\delta=\sum_{j=1}^{2} c_{j} m_{j} \xi / \rho_{j},
$$

where $\rho_{1}$ and $\rho_{2}$ are the densities of yttrium and titanium, i.e. $4.47 \times 10^{3} \mathrm{~kg} / \mathrm{m}^{3}$ and $4.50 \times 10^{3}$ $\mathrm{kg} / \mathrm{m}^{3}$, respectively.

\subsection{Measurement of molar ratio between film components}

RBS can be used to measure the molar ratio between film components. The total number of incident alpha particles is denoted as $Q$. When they are scattered from the nuclei of component $j$, the number recorded by the detector of RBS is denoted as $\delta Q_{j}$. The atomic surface density of this component can be written as 21$]$

$$
\xi_{j}=\frac{\delta Q_{j}}{Q \cdot d \sigma_{j}}
$$

where $d \sigma_{j}$ is the Rutherford backscattering cross-section.

The molar ratio between two components of a film is equal to the ratio of their atomic surface densities. Substitution of the expression of $d \sigma_{j}$ gives

$$
\frac{c_{1}}{c_{2}}=\frac{\delta Q_{1} d \sigma_{2}}{\delta Q_{2} d \sigma_{1}}=\frac{m_{1} Z_{2}^{2} \delta Q_{1}}{m_{2} Z_{1}^{2} \delta Q_{2}} \times \frac{\sqrt{m_{1}^{2}-m_{\alpha}^{2} \sin ^{2} \vartheta}}{\sqrt{m_{2}^{2}-m_{\alpha}^{2} \sin ^{2} \vartheta}} \times\left[\frac{\sqrt{m_{2}^{2}-m_{\alpha}^{2} \sin ^{2} \vartheta}+m_{2} \cos \vartheta}{\sqrt{m_{1}^{2}-m_{\alpha}^{2} \sin ^{2} \vartheta}+m_{1} \cos \vartheta}\right]^{2} .
$$

In ICP-AES measurement, the film is firstly dissolved with hydrochloric acid, and then the solution is sprayed and further vaporized, dissociated and excited in a high-temperature plasma. 
The molar contents of the film can be obtained from the intensities of the characteristic emission spectra of the excited atoms or ions ${ }^{[23]}$.

\section{DSMC calculations}

The DSMC method employs a large number of simulation molecules to represent actual molecules in a gas flow. Their physical information such as positions and velocities is stored in a computer, and changes with time due to transport processes, such as molecular motion and collisions, and interaction with boundaries. The nature of DSMC is to decouple the molecular movement and collisions in a small time interval. In a time step $\Delta t$, all molecules move a distance according to their velocities, and then the representative collisions between molecules in the time step are calculated. In order to enable such a time decoupling to reflect the essential characteristics of real molecular motion in a statistical way, $\Delta t$ should be less than the local mean collision time. The collision pairs are chosen in a cell, and the cell is also used for sampling and for averaging the molecular information statistically to obtain macroscopic quantities, such as the velocity, number density and temperature fields.

\subsection{Computational domain}

Our computational domain is a circumscribed cuboid $(760 \mathrm{~mm} \times 760 \mathrm{~mm} \times 365 \mathrm{~mm})$ of the vacuum chamber (Figure 1) between the evaporation and the substrate planes. Uniform grids are used in directions $X$ and $Y$ with grid number of 60. Non-uniform grids are used in the $Z$ direction with grid number of 40 , where the grid size will be refined locally and the size of those grids adjacent to the evaporation plane is $1 \mathrm{~mm}$.

The number density of vapor atoms around the evaporation sources is large, and the frequent collisions between them affect considerately their movement trajectories. The mean collision time of the particles adjacent to the evaporation sources ranges between $2 \times 10^{-5} \mathrm{~s}$ and $4 \times 10^{-5} \mathrm{~s}$. Therefore, the time step in DSMC is taken to be $1 \times 10^{-5} \mathrm{~s}$ to satisfy the time step requirement for decoupling the movement and collision in the DSMC method.

\subsection{Boundary conditions and collision model}

The side wall of the vacuum chamber water-cooled keeps a temperature of $293 \mathrm{~K}^{[18]}$, and a non-heated substrate has the same temperature as the chamber wall. Because the surface temperatures of the chamber and substrate are much lower than the melting points of yttrium and titanium, the sticking coefficients are approximately one as their vapor atoms strike onto the surfaces. Therefore, the boundaries except for the evaporation sources may be treated as completely absorbable in DSMC calculations.

In a time step $\Delta t$, the number of the vapor particles entering the computational domain from an evaporation source $\ell$ can be expressed as ${ }^{[7,8]}$

$$
N_{\ell}=\gamma n_{\ell} \cdot \sqrt{k T_{\ell} / 2 \pi m_{\ell}} \cdot A_{\ell} \cdot \Delta t,
$$

where $\gamma$ is the ratio of simulated particles to real ones, and $A_{\ell}=\pi d_{e}^{2} / 4$. In our calculations, the mean number of simulation particles in a cell is about 20 depending on the selection of $\gamma$, and their total number is about $3 \times 10^{6}$.

The initial velocities of vapor atoms are determined based on Maxwellian distributions. The evaporation source surfaces are completely diffusely reflective so that the calculated and experi- 
mental evaporation masses are the same $e^{[6]}$. The background pressure in the vacuum chamber during the experiments keeps about $1 \times 10^{-3} \mathrm{~Pa}$, and the molecules of the background gases have negligible effect on the trajectories of the vapor atoms, which are thus ignored in the calculations.

The VHS model ${ }^{[10]}$ is adopted to describe the interaction between vapor atoms, and the reference collision diameters and power exponents of VHS for yttrium and titanium vapor atoms come from Table II in ref. [6].

\subsection{Computational method of film thickness and molar ratio}

The deposition thickness of yttrium and titanium vapor atoms onto the surfaces of a quartz crystal resonator or silicon wafer may be calculated as

$$
\delta=t_{e} \sum_{j=1}^{2} m_{j} n_{j}^{p} v_{j, \perp}^{p} / \rho_{j},
$$

where $n_{j}^{p}$ and $v_{j, \perp}^{p}$ are the number density and normal velocity of component $j$ at these surfaces, respectively.

The surface of a silicon wafer is assumed to be completely sticking, so the molar ratio between components in the yttrium-titanium alloy film is

$$
\frac{c_{1}}{c_{2}}=\frac{n_{1}^{p} v_{1, \perp}^{p}}{n_{2}^{p} v_{2, \perp}^{p}} .
$$

\section{Comparison of computational and experimental results}

\subsection{Case I: co-evaporation with two sources}

Figure 4 presents contours of the number density and velocity component in the $Z$ direction in a jet flow field with two evaporation sources. Similar to the yttrium jet with single evaporation source reported in ref. [6], the vapor jet expands rapidly under the vacuum condition, and the number density decreases about $1-2$ orders in magnitude within a distance of 1 inch after leaving the evaporation surface while the vapor velocity reaches $700 \mathrm{~m} / \mathrm{s}$. When the jet arrives at the substrate, it experiences a full expansion, as a result the number density drops to about four orders lower than that at the evaporation surface. Different from ref. [6], the velocity firstly increases and then decreases along the normal direction of the titanium evaporation source, instead of a monotonic increase. The reason is that the atomic weights of yttrium and titanium are 48 and 89 , respectively, and thus after the expansion the speed of the latter is significantly lower than the former, resulting in a velocity decline of the mixture vapors far from and perpendicular to the yttrium evaporation source.

The mean evaporation rates of yttrium and titanium (Figure 2) measured with quartz crystal resonators $P 1$ and $P 2$ are 2.04 and $2.02 \AA / \mathrm{s}$, respectively, while those given by the DSMC calculation using eq. (11) are 1.94 and $1.97 \AA / \mathrm{s}$. They are found in good agreement.

In this case, the origin of a 4-inch silicon wafer is located exactly above the midpoint of $G 1$ and $G 2$ (Figure 1). The crystal symbol is close to $G 1$, while the shield slices are parallel to the $Y$ direction. Figure 5 shows 18 positions for measuring the film thickness and the molar ratio after the experiment. RBS measurements are carried out in all these positions, profilometer measurements are performed in 12 of them (except 3, 4, 9, 10, 15 and 16), and ICP-AES measurements in 4 of them (1, 5, 8 and 17). Typical measured curves of RBS and ICP-AES are shown in Figures 6 and 7. 

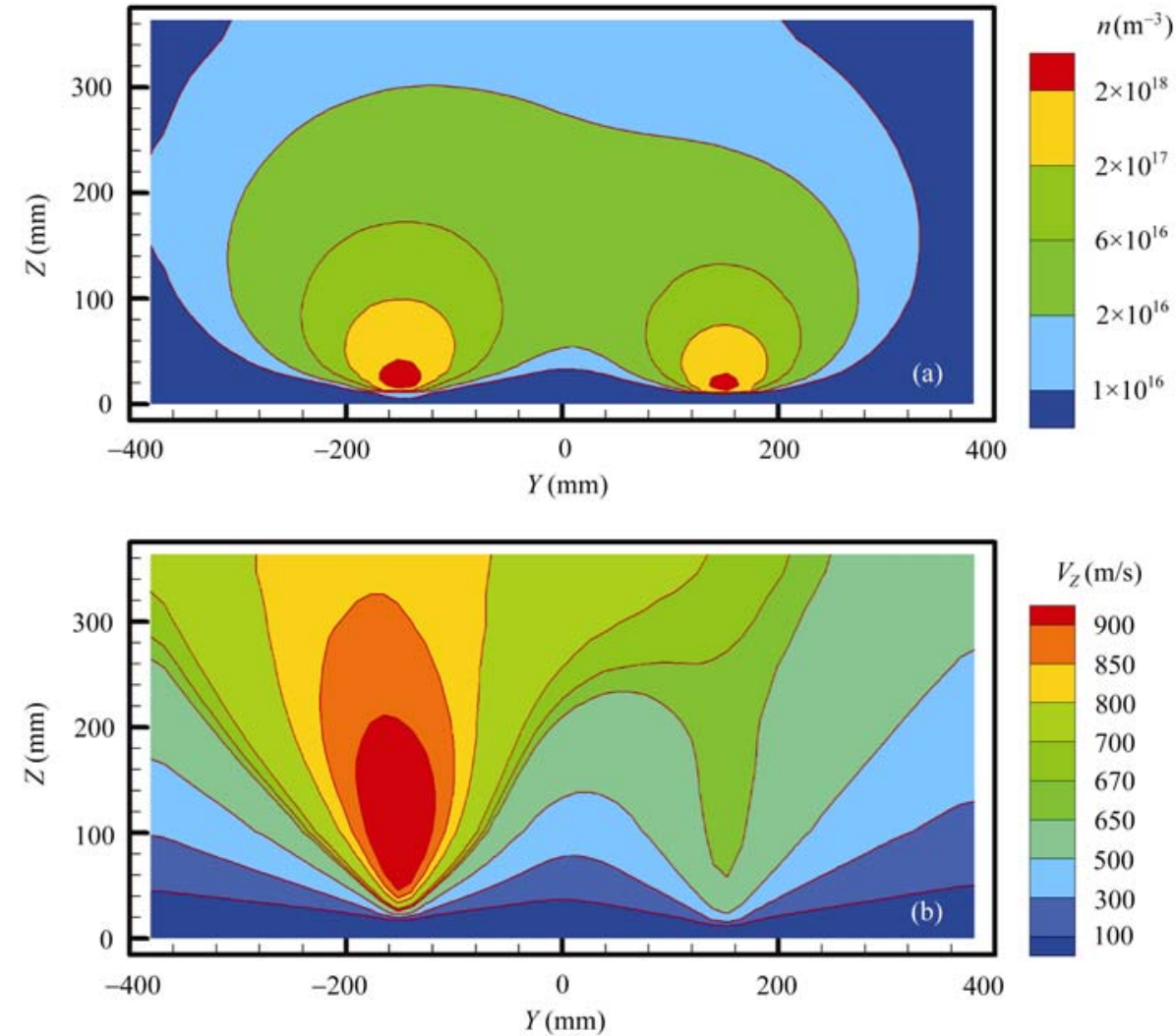

Figure 4 Flow field contours of yttrium and titanium mixture vapors given by DSMC for Case I. (a) Number density; (b) velocity component in the direction perpendicular to the wafer surface.

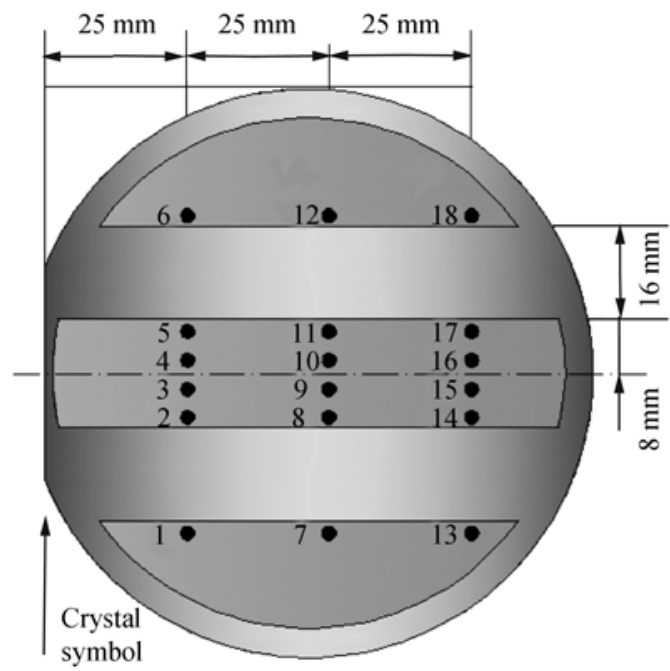

Figure 5 Positions for measuring the film thickness and molar ratio over a 4-inch silicon wafer in Case I.

The calculated and experimental values of the yttrium-titanium film thickness distribution over the 4-inch wafer are compared in Figure 8. The difference between DSMC and RBS results is about $7 \%$ at most and $3 \%$ on average. The mean value of the 12 data of the profilometer measurements is about $2389 \AA$, while the corresponding value given by DSMC is $2357 \AA$, which is only $2 \%$ lower than the former. 


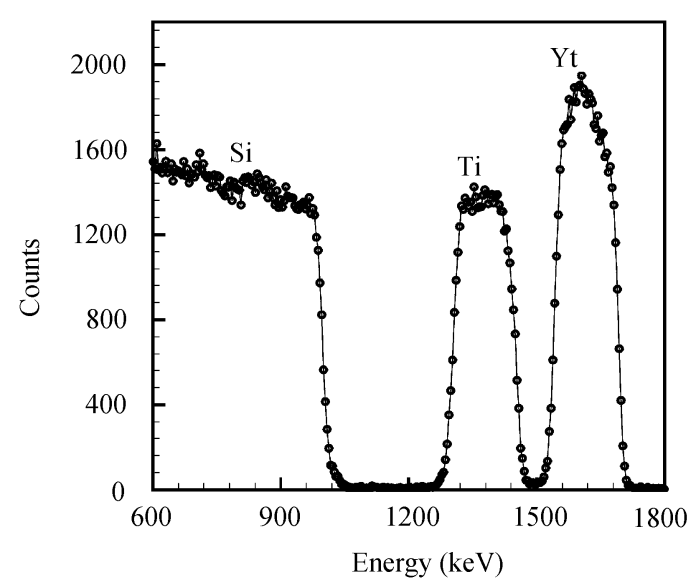

Figure 6 RBS energy spectrum at measurement position 8 in Case I.
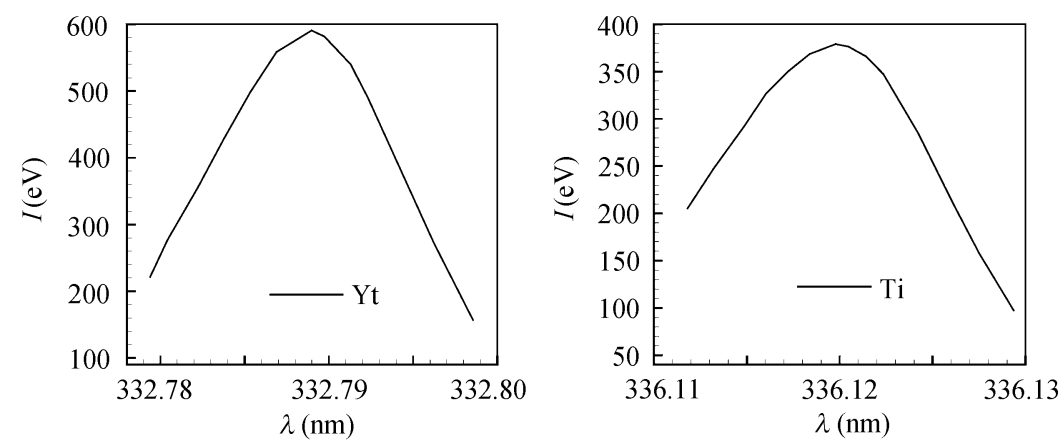

Figure 7 ICP-AES spectrum at measurement position 1 in Case I.

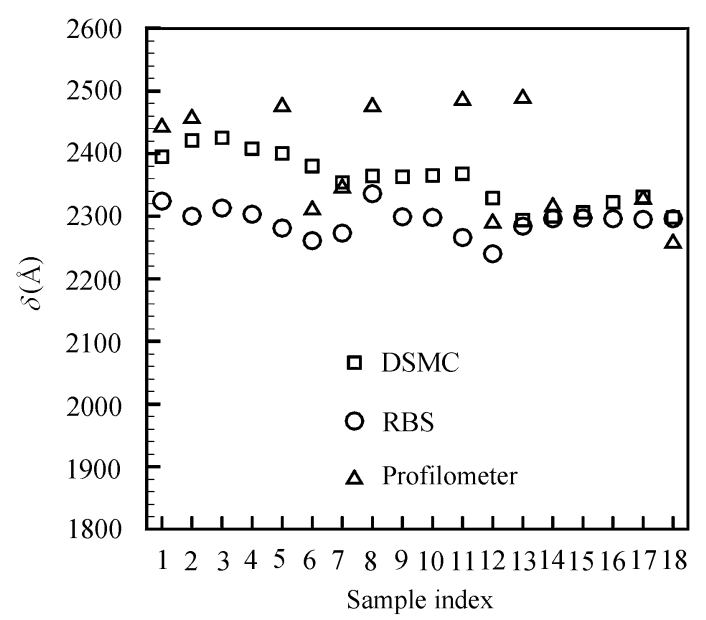

Figure 8 Yt-Ti film thickness distributions over a 4-inch wafer given by DSMC, RBS and Dektak8 for Case I.

Because the substrate in our experiments is kept at room temperature, the diffusive capability of the vapor particles on its surface is weak. Due to the statistical fluctuation and the shadow effect, there are defects and holes occurring during the film growth ${ }^{[24]}$. The densities of the yttrium and titanium components in the films are thus less than those of the ingots. This makes the film thickness calculated by eq. (11) slightly lower than the measured one by the surface profilometer. 
Furthermore, the coarse film surfaces lead to significant scattering in the measurement given by surface profilometer.

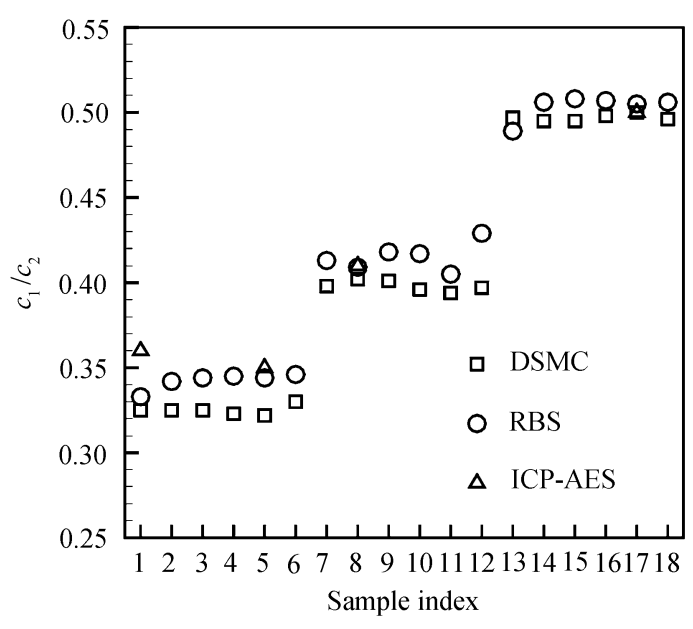

Figure 9 Molar ratios of component yttrium to titanium over a 4-inch wafer given by DSMC, RBS and ICP-AES for Case I.
Figure 9 shows molar ratios between the yttrium and titanium components at 12 places on the film given by DSMC method, and they are compared to the measured data of RBS and ICP-AES. Both measured data agree very well with each other, and the difference is only $2 \%$ on average. The DSMC results are also found in good agreement with experimental data: the maximum difference is about $5 \%$ and takes place at position 12. It is noted that the DSMC results are obtained prior to the RBS and ICP-AES measurements, thus highlighting the ability of the DSMC approach developed here in predicting a practical process.

Figure 10 presents the contours of the film thickness and the molar ratio of the component titanium to yttrium over the 4-inch silicon wafer as given by DSMC method. Because the evaporation mass of titanium is slightly greater than that of yttrium, the film thickness monotonically decreases about $5 \%$ in magnitude in the $Y$ direction. The atomic weight of titanium is about two-times lighter than that of yttrium, and consequently the molar ratio varies greatly, with the biggest one 2.3 times of the least one.
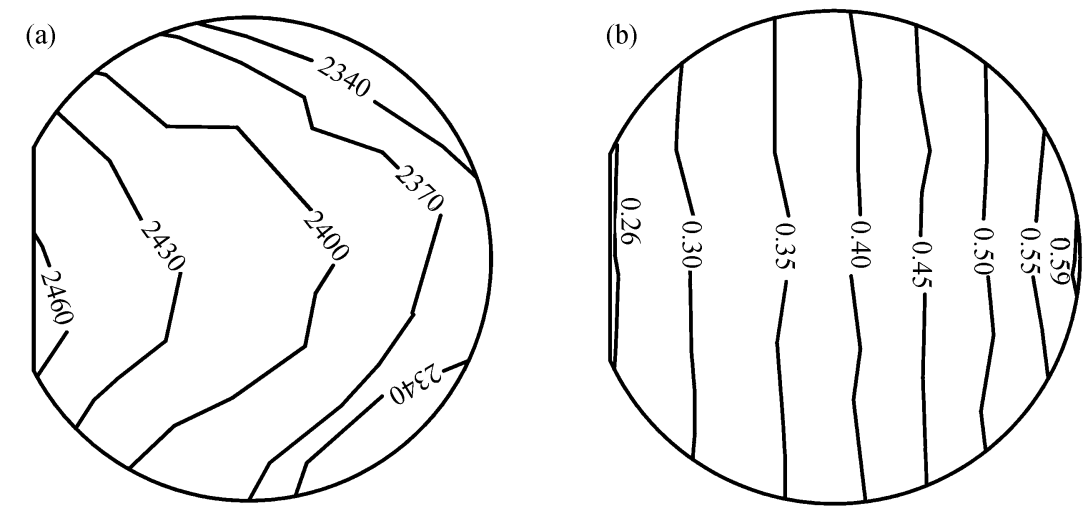

Figure 10 Yttrium-titanium film distributions over a 4-inch wafer given by DSMC for Case I. (a) Thickness ( $\AA$ ); (b) molar ratio of component yttrium to titanium.

\subsection{Case II: co-evaporation with three sources}

Figure 11 shows the number density and the velocity component in $Z$ direction of the mixture vapors evaporating from three sources in Case II given by DSMC method. Compared with the two-source case shown in Figure 4, the three-source flow field is more complicated, and therefore it is difficult and time-consuming to optimize technological parameters of co-deposition with multi-sources by traditional method of trial and error. 

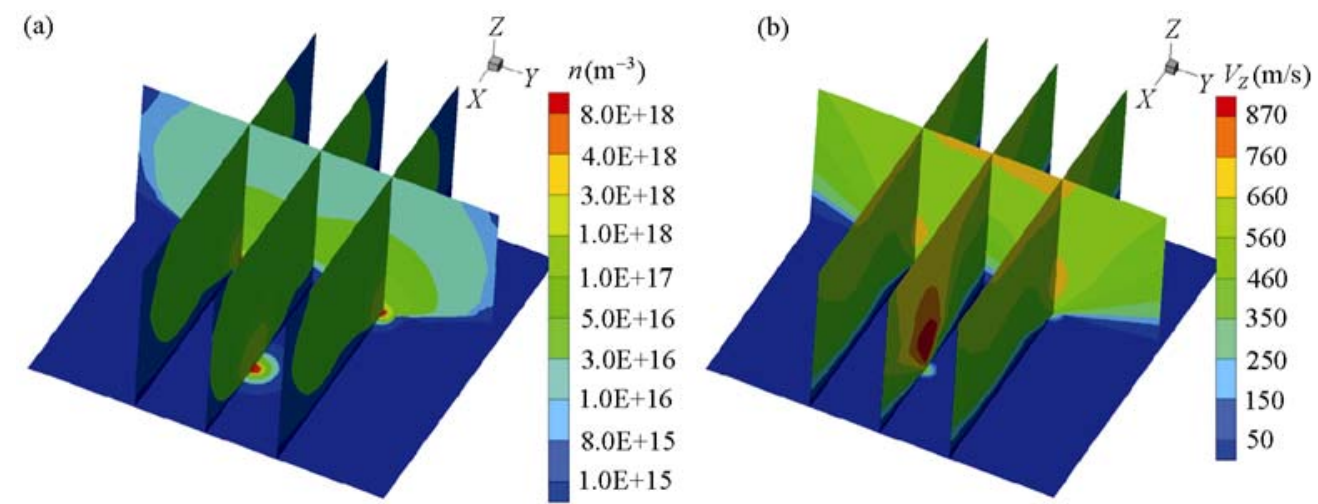

Figure 11 Flow field contours of yttrium and titanium mixture vapors given by DSMC for Case II. (a) Number density; (b) velocity component in the direction perpendicular to the wafer surface.

The center of a 6-inch mono-crystal silicon wafer is exactly above the origin $O$ (Figure 1), and the crystal symbol is close to $G 3$. The shield slices are parallel to the $X$ axis. Figure 12 presents the film thickness distribution obtained by DSMC calculation, which agrees quite well with measurements given by the surface profilometer shown in Figure 4. The mean film thickness of DSMC over the whole wafer is $2395 \AA$, while the mean value of experimental data at the 20 positions is $2437 \AA$. The relative difference between them is $-2.1 \%-+2.4 \%$ only. This is easily understood because the equilateral triangular arrangement of the three evaporation sources favors the uniformity of the vapor particles flux through the wafer surface.

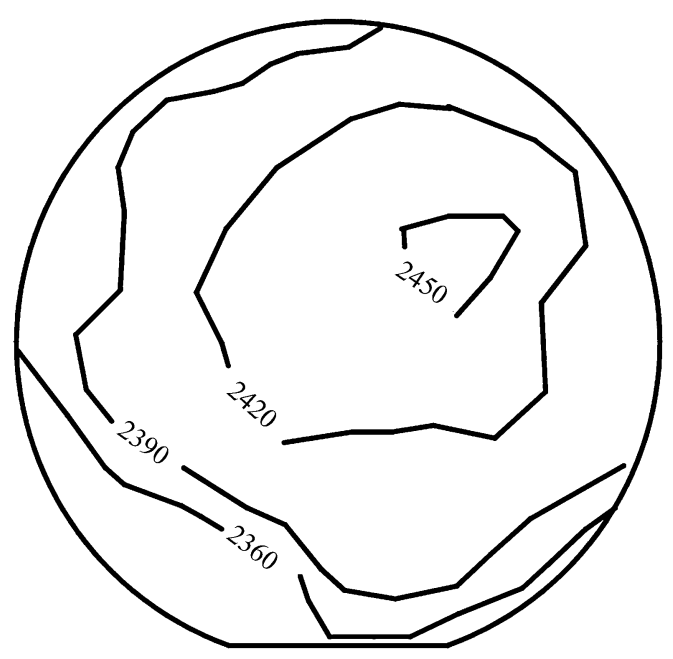

Figure 12 Yttrium-titanium film thickness $(\AA)$ distribution over a 6-inch wafer given by DSMC for Case II.

\section{Conclusions}

The experimental and numerical approaches are combined to analyze in details the low-density, non-equilibrium jets of yttrium and titanium vapors in EBPVD, and the distributions of deposited thickness and molar contents of the vapor particles over the mono-crystal silicon wafers. The numerical results of DSMC method are found in excellent agreement with experimental data obtained via four different measurement methods. The detailed flow fields provided by DSMC are 
helpful for us to understand the statistical features of the transport trajectories of vapor particles from the evaporation sources to the wafer surfaces in physical vapor deposition, which may pave the way for an economical and quick approach to optimize the co-deposition technique of large-area, multi-component films.

Material design and fabrication at the atomic level is an important goal of modern materials science. To achieve such a goal in EBPVD, we need to establish quantitative relations between atomic motions and technological conditions in three processes, i.e. the interaction process between atoms at evaporation ingot surfaces and impinging high-energy electrons, the transport process of vapor atoms in a vacuum chamber, as well as the diffusion and condensation process of vapor atoms on a wafer surface. The present work builds up certain relations for the transport process, which also provides accurate boundary conditions for quantitative investigation of the film growth process. In further study, emphases will be laid on atomic motions at the vapor-solid interface of a wafer, in particular relationship between macroscopic conditions, such as the wafer temperature, incident velocity of vapor particles, etc, and film growth characteristics.

1 Wang E G. Atomic-scale study of kinetics in film growth (I). Prog Phys (in Chinese), 2003, 23(1): 1-61

2 Holland L, Steckelmacher W. The distribution of thin films condensed on surfaces by the vacuum evaporation method. Vacuum, 1952, 2: 346-364

3 Behrndt K H. Thickness uniformity on rotating substrates. In: Bancroft G H, ed. Transactions of 10th National Vacuum Symposium of the American Vacuum Society, Boston, USA. New York: Macmillan Co., 1963, 379-384

4 Graper E B. Distribution and apparent source geometry of electron-beam-heated evaporation sources. J Vac Sci Tech, 1973, 10(1): $100-103$

5 Powell A, Minson P, Trapaga G, et al. Mathematical modeling of vapor plume focusing in electron beam evaporation. Metal Mater Trans A, 2001, 32(8): 1959-1966[DOI]

6 Fan J, Boyd I D, Shelton C. Monte Carlo modeling of electron beam physical vapor deposition of yttrium. J Vac Sci Tech A, 2000, 18(6): 2937-2945[DOI]

7 Bird G A. Molecular Gas Dynamics and the Direct Simulation of Gas Flows. Oxford: Clarendon Press, 1994

8 Shen C. Rarefied Gas Dynamics: Fundamentals, Simulations and Micro Flows. Berlin: Springer, 2005

9 Bird G A. Molecular Gas Dynamics. Oxford: Clarendon Press, 1976

10 Bird G A. Monte Carlo simulation in an engineering context. In: Fisher S S, ed. The 12th International Symposium on Rarefied Gas Dynamics, Charlottesville, USA. New York: American Institute of Aeronautics and Astronautics, 1981. $239-255$

11 Koura K, Matsumoto H. Variable soft sphere molecular model for inverse-power-law or Lennard-Jones potential. Phys Fluids A, 1991, 3(10): 2459-2465[DOI]

12 Hassan H A, Hash D B. A generalized hard-sphere model for Monte Carlo simulated. Phys Fluids A, 1993, 5(3): $738-744[\mathrm{DOI}]$

13 Fan J. A generalized soft-sphere model for Monte Carlo simulation. Phys Fluids, 2002, 14(12): 4399-4405[DOI]

14 Chapman S, Cowling T G. The Mathematical Theory of Non-Linear Gases. 3rd ed. Cambridge: Cambridge University Press, 1970

15 Balakrishnan J, Boyd I D, Braun D G. Monte Carlo simulation of vapor transport in physical vapor deposition of titanium. J Vac Sci Tech A, 2000, 18(3): 907-916[DOI]

16 Hass D D, Marciano Y, Wadley H N G. Physical vapor deposition on cylindrical substrates. Surf Coat Tech, 2004, 185: $283-291[\mathrm{DOI}]$

17 Fan J, Boyd I D, Shelton C. Monte Carlo modeling of YBCO vapor deposition. In: Bartel T J, Gallis M A, eds. The 22nd International Symposium on Rarefied Gas Dynamics, Sydney, Australia. New York: Melville. 2001, 214-221

18 Fan J, Shu Y H, Liu H L, et al. Development report of multi-source electron beam physical vapor deposition (in Chinese). IMCAS STR 2005003. Institute of Mechanics, Chinese Academy of Sciences, Beijing, China, 2005

19 Dushman S. Scientific Foundation of Vacuum Technology. New York: John Wiley \& Sons, 1962

20 Lu C S. Mass determination with piezoelectric quartz crystal resonators. J Vac Sci Tech, 1974, 12(1): 578-583

21 Chu W K, Mayer J W, Nicolet M A. Backscattering Spectrometry. New York: Academic Press, 1978

22 Ziegler J F. Helium-stopping powers and ranges in all elements. In: The Stopping and Ranges of Ions in Matter. New York: Pergamon Press, 1977

23 Sun H W. Atomic Spectroscopy Analysis (in Chinese). Beijing: Higher Education Press, 2002

24 Thornton J A. High Rate Thick Film Growth. Ann Rev Mater Sci, 1977, 7: 239-260 